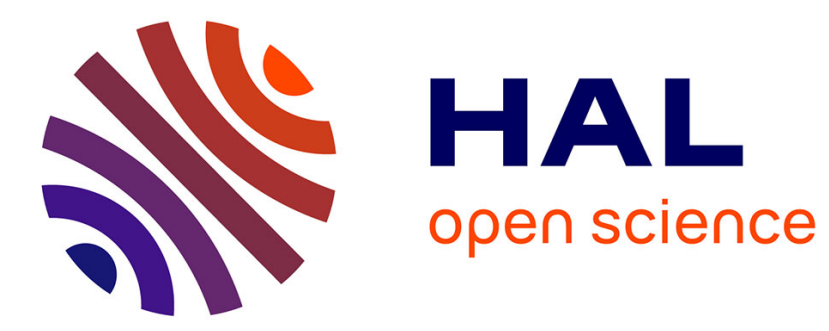

\title{
Supervising SSSEP experiments with a Bluetooth Android remote control application
}

José Rouillard, François Cabestaing, Jean-Marc Vannobel, Marie-Hélène Bekaert

\section{- To cite this version:}

José Rouillard, François Cabestaing, Jean-Marc Vannobel, Marie-Hélène Bekaert. Supervising SSSEP experiments with a Bluetooth Android remote control application. International Conference on Applied Human Factors and Ergonomics, Jul 2018, Orlando, United States. 10.1007/978-3-319-948669_37. hal-01915111

\author{
HAL Id: hal-01915111 \\ https://hal.science/hal-01915111
}

Submitted on 7 Nov 2018

HAL is a multi-disciplinary open access archive for the deposit and dissemination of scientific research documents, whether they are published or not. The documents may come from teaching and research institutions in France or abroad, or from public or private research centers.
L'archive ouverte pluridisciplinaire HAL, est destinée au dépôt et à la diffusion de documents scientifiques de niveau recherche, publiés ou non, émanant des établissements d'enseignement et de recherche français ou étrangers, des laboratoires publics ou privés. 


\title{
Supervising SSSEP experiments with a Bluetooth Android remote control application
}

\author{
José Rouillard $^{1}$, François Cabestaing ${ }^{1}$, Jean-Marc Vannobel ${ }^{1}$, Marie-Hélène Bekaert ${ }^{1}$ \\ ${ }^{1}$ Univ. Lille, CNRS, Centrale Lille, CRIStAL, UMR 9189, \\ 59655 Villeneuve d'Ascq Cedex, France \\ \{Jose.Rouillard, Francois.Cabestaing, Jean-Marc.Vannobel, Marie-Hélène.Bekaert\} \\ @ univ-lille1.fr
}

\begin{abstract}
In this paper, we are presenting how we are controlling the vibration frequency sent from an Android smartphone to an Arduino board connected to a vibrator, in order to supervise more easily SSSEP (Steady State Somatosensory Evoked Potentials) experiments. Our researches are conducted in the context of hybrid Brain-Computer Interfaces for motor severely impaired patients, and our aim is to detect a physiological gating phenomenon on SSSEP responses when patients are trying to perform some small fingers moves while vibrations are emitted under their fingers.
\end{abstract}

Keywords: Hybrid BCI · SSSEP · Gating · Vibration · Smartphone · Arduino

\section{Introduction}

The concept of Brain-Computer Interface (BCI), introduced by Vidal [1] in 1973, is a system that allows users to communicate between human's brain and external devices. Researchers are investigating different kinds of paradigm related to BCI (with or without stimuli, etc.) and several manners to measure the human activity during invasive or non-invasive BCI experiments (ElectroEncephaloGraphy -- EEG, ElectroMyoGraphy - EMG, MagnetoEncephaloGraphy - MEG, etc.). They are also developing various kinds of BCI solutions according to different users (healthy or disable people, etc.). To improve the speed and robustness of communication, Leeb et al. have introduced the so-called "hybrid BCI" notion, in which brain activity and one or more other signals are analyzed jointly [2].

Since BCI is considered as an effective tool for rehabilitation and/or assistance of severely impaired patients, we are particularly working in our research team on hybrid BCI for Duchenne Muscular Dystrophy (DMD). DMD is a severe pathology of the skeletal musculature. This genetic disorder causes an absence of dystrophin, a protein that supports muscle strength and muscle fibers cohesion, which leads to progressive muscle degeneration and weakness. The residual motor ability of the most advanced DMD patients is characterized by very low amplitude movements associated with loss 
of degrees of freedom and severe muscle weakness in the fingers. Specific brain activities can be detected in these patients. For instance, an event-related potential (ERP) is a measured brain electrophysiological modification, response to either an external stimulation (sound, image, vibration, etc.) or an internal event such as a cognitive activity (attention, motor preparation, etc.) [3].

Since the first recordings of electrical activity from the human brain, many studies have reported ERP as changes in brain signal expressing responses to sensory stimuli. Instead of observing sudden and time-lock responses to a transient event (for instance a P300 signal occurs $300 \mathrm{~ms}$ after a stimulus), it is also possible to detect more steady-state evoked potentials (SSEP). "An increasing number of studies have used SS-EP to explore the neural activity involved in the cortical processing of visual and auditory sensory modalities and, to a lesser extent, the somatosensory modality" [4].

Part 2 of the paper presents the background of this research within the BCI field and a state of the art concerning Steady State Evoked Potentials. Part 3 describes our contribution and methods to explore this field of study. The results, conclusion and perspectives are presented in part 4.

\section{Steady State Evoked Potentials and BCIs: a brief overview}

Steady state visually evoked potentials (SSVEP) are signals that are natural responses to visual stimulation at specific frequencies. Indeed, when the retina is excited by a visual stimulus $(3.5 \mathrm{~Hz}$ to $75 \mathrm{~Hz})$ the brain generates electrical activity at the same (or harmonic) frequency of the visual stimulus. Electrodes are positioned mainly on the occipital part of the human scalp in order to detect SSVEP. For example, BCIs used SSVEP to control a computer cursor [5][6], an avatar [7], a robot [8][9], a wheelchair [10] or a spelling system [11][12].

Steady state auditory evoked potential (SSAEP) can be used to trace the signal generated by a sound (often at a frequency between 5 and $50 \mathrm{~Hz}$ ) through the ascending auditory pathway when an evoked potential is generated from the cochlea to the cortex. Electrodes are positioned mainly on the temporal part of the human scalp in order to detect SSAEP. Steady state auditory evoked potential can be used to replace steady state visual evoked potential in brain-computer interface systems during visual fatigue periods [13]. Auditory ERP can also be used in auditory speller BCI or multichoice based BCI [14][15].

Steady state somatosensory evoked potentials (SSSEP) are detected as cerebral responses to vibratory stimulation applied on the body of the user (palm of the hand, wrist, finger, toe) with frequencies in the range of 5-250 Hz. Electrodes are positioned accordingly, for instance in C3, C4, or $\mathrm{Cz}$ location (see 10/20 international system) to detect a brain signal response to a vibration applied on right finger, left finger or toe, respectively. SSSEP based BCIs may reduce the fatigue usually induced by visual attention required in SSVEP based BCIs. Steady state somatosensory evokes potential are used for communication tools dedicated in complete locked-in syndrome (CLIS) patients for which SSVEP are inoperative [16]. The feasibility of SSSEP 
based BCIs for wheelchair control [17][18], or task discrimination BCIs is also studied [19].

The part of the somatosensory system that transmits pain and temperature signals is monitored using laser evoked potentials (LEP) [20][21]. Colon et al. explored the possibility to use SSEP in response to the thermal activation of cutaneous nociceptors in humans: "Recently, we showed that it is possible to record SSEP in response to the rapid periodic thermal activation of cutaneous nociceptors in humans, as well as to the rapid periodic electrical stimulation of nociceptive intraepidermal free nerve endings" [4].

Steady-state somatosensory evoked potential (SSSEP) can be produced by vibratory stimulation. Transducers provide different tactile stimulations in the resonance-like frequencies of the sensorymotor areas [22][23]. Stimuli were applied on different parts of the body (e.g. palm of the hand, wrist, finger, toe) so that the user had to focus his attention either to one or other part. The increase of one of the elicited SSSEPs amplitude was detected in EEG signals [24].

In 2016, Sangtae Ahn and al. [25] draw up a complete state of the art on SSSEP for BCI. After Müller-Putz and al. [24] who first defined the basic SSSEP-based BCI paradigm with index fingers stimulations, several studies on SSSEP based BCI have been published. Some stimulate the thumbs to discriminate between the two hands [26]. Breitwieser et al. [27] stimulate all the fingers of the right hand. In addition to finger stimulation, Yao et al. [28] applied stimulation on the skin of both wrists. Ahn et al. [29] performed a hybrid BCI based on motor imaging and SSSEP. This study demonstrated better classification performance in motor imaging using selective touch attention. These studies show the feasibility of BCIs based on the SSSEP but the performances achieved so far are quite low.

Otherwise, appears a phenomenon called "gating" or tactile suppression describing an attenuation of somatosensory input to the cerebral cortex during movement execution [30][31]. This gating seems to be detected not only when users are moving while feeling a vibration under their fingers, but also when users are watching video showing hands moving or being touched by a virtual object: "Observation of passive touch of the hand also gated the response (17\% decrease). In conclusion, the results show that viewing a hand performing an action or being touched interferes with the processing of somatosensory information arising from the hand". [32]. It seems that the "gating" phenomenon has not yet been used in BCI studies.

\section{Methods}

We try to exploit this physiological phenomenon within a BCI based on the SSSEP paradigm. Korean researchers have shown that a wheelchair can be driven by users asked to focus on certain vibrations emitted on left wrist, right wrist or toe, through SSSEP (with 30 electrodes) [18];

Canadian researchers have demonstrated that visualization by a subject of a video showing a movement of the hand, while some vibrations are sent to this patient's fin- 
ger, interferes with the somatosensory process of information recovery for, in average, an amplitude reduction of 22\% of SSSR (somatosensory steady-state response) [32] ; We assume that it would be possible to use this physiological gating in a hybrid BCI as an interaction command.

We want to check if the following hypothesis is correct or not: by placing two vibrators under the left and right fingers of a DMD subject and by asking him to perform small finger movements, should we observe a decrease in the SSSR between 10 to $20 \%$ at the determined frequency (e.g. 7 or $13 \mathrm{hz}$ ) at C3 and C4 (and maybe on a single Cz-electrode), and therefore, infer that the user moved (or wanted to move) to the right or left direction?

This preliminary study on healthy user (see figure 1,3 ) will be the first step to achieve a solution where the DMD patient will only think about the finger movement without really performing it.

\subsection{Overall architecture}

As presented in figure 1, our overall architecture is organized in order to facilitate BCI experiments based on the acquisition of cerebral signals during SSVEP studies.

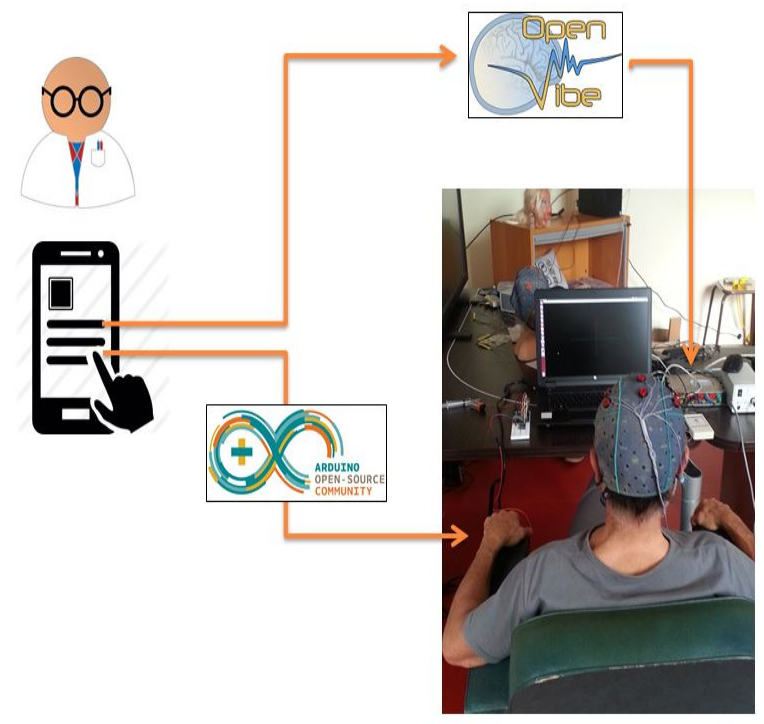

Fig. 1. Our overall architecture for the study of SSSEP.

The experimenter chooses a frequency (for instance $12 \mathrm{~Hz}$ ) thanks to the mobile Android smartphone application that we have developed in our team. This frequency is sent by BLE (Bluetooth Low Energy) to an Arduino board connected to a vibrator device. At the same time, this frequency is also sent, via UDP, to the software in 
charge of the cerebral signals detection (OpenVibe), which can be found in an EEG when sending vibrations under the fingers or wrists of patients.

\subsection{Technical aspects}

\section{Mobile Application}

Our mobile Android prototype application was developed with App inventor 2 which is a tool proposed jointly by Google and the MIT. We injected in this application both "BluetoothLE" extension [33] and "ClientUDP" extension [34] in order to communicate respectively through BlueTooth Low Energy protocol and User Datagram Protocol.

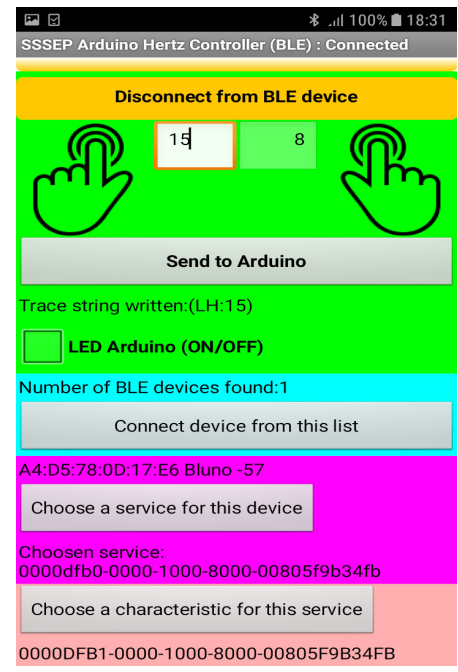

Fig. 2. Prototype of mobile application used in our SSSEP studies.

As we can see in figure 2, this mobile application prototype can be easily used by experimenters to enter the desired frequency and to send it wirelessly to the Arduino board and the BCI software.

\section{Arduino based vibratory stimulator}

Linear Resonant Actuators (LRA) as shown in figure 3 are nowadays commonly used instead of eccentric rotating mass (ERM) motors for cell phones to vibrate. That makes them easy to find and affordable. A LRA can be compared to a miniaturized loud speaker making a round metallic pad moving from the front to the back instead of a cone. LRAs are then really convenient to use in haptic applications and best used within a narrow frequency vibration range centered around a resonant frequency close to $200 \mathrm{~Hz}$ (precisely $205 \mathrm{~Hz}$ for the $10 \mathrm{~mm} \mathrm{C10-000} \mathrm{LRA} \mathrm{presented} \mathrm{in} \mathrm{figure} 3$ ). 

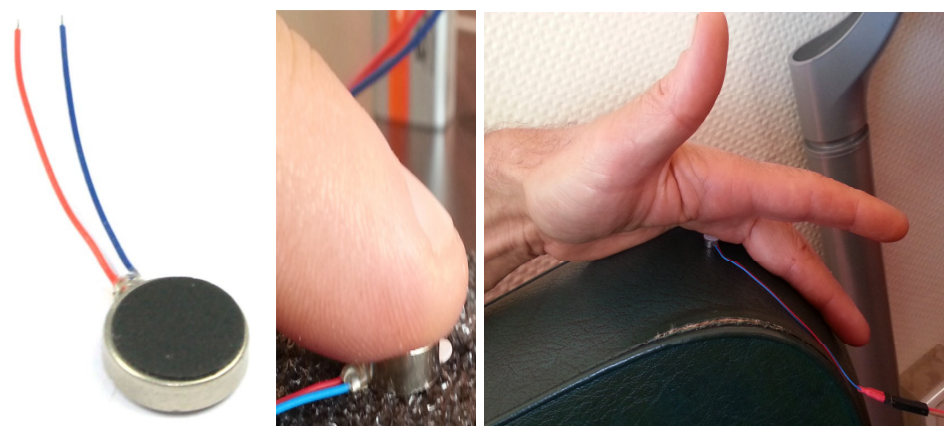

Fig. 3. LRA vibrator (left) applied under the finger (center) or under the hand palm (right).

An Adafruit DRV2605 board as shown in figure 4 (right) has been used to power up a 10mm C10-000 LRA. This board is based on a TI DRV2605 motor driver [35]. As shown in figure 4 (left), an I2C (Inter Integrated Circuit) communication can be used to manage this driver from the vibration frequency control board. However, one has to note that the DRV2605 does not have I2C address pins neither a chip select one. To control more than one driver through $\mathrm{I} 2 \mathrm{C}$ communication one have then to implement as much I2C communication buses as DRV2605 drivers used or to multiplex the existing I2C communication bus.

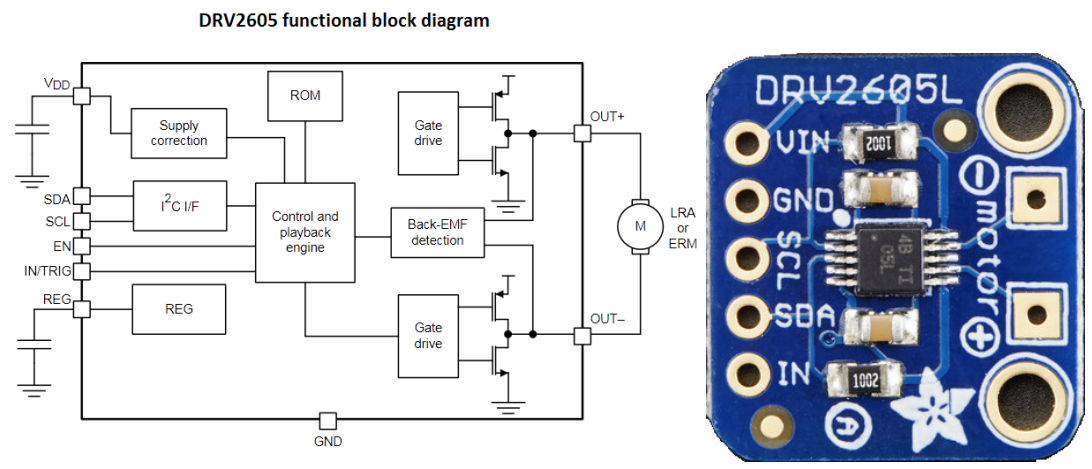

Fig. 4. TI DRV2605 driver functional block diagram (from [10], left) and Adadruit DRV2605 board (right).

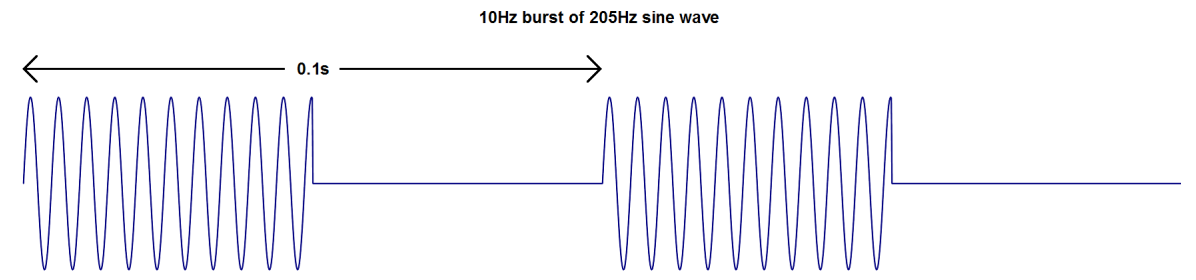

Fig. 5. TI DRV2605 $10 \mathrm{~Hz}$ burst of $205 \mathrm{~Hz}$ sine wave output. 
As previously said, an Arduino based board was used to control the low frequency burst of $205 \mathrm{~Hz}$ sine wave, as illustrated in figure 5, applied to the C10-000 LRA. Both BLE and I2C communication were needed. We could then use either an Arduino UNO and a BLE shield as the RedbearLab one for example (figure 6, left) or better a DFRobot Bluno Beetle board (figure 6, center) which offers an Arduino with Bluetooth Low Energy communication interface. In short, the Bluno Beetle is fully compatible with the Arduino Uno board except the number of inputs and outputs found in reduced quantity, uses the original Arduino IDE without the need of any external library for Bluetooth communication and can be used with any Bluetooth 4.0 compatible device.

At last, we present figure 6 (right) the whole development unit used in this haptic application. As one can see, this is really simple to implement since needing only a Bluno Beetle microcontroller board, a DRV2605 driver to drive a LRA and the LRA itself.

In haptic applications needing information coming from both hands, two LRA drivers and LRAs are of course needed. As previously said, one has then to take into account that a second I2C communication interface is needed. A software one can easily be computed in that way.
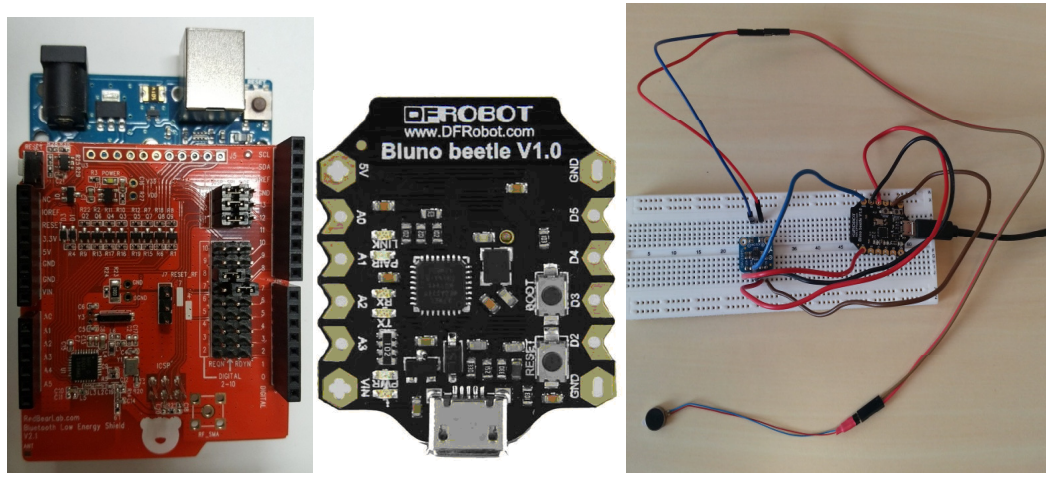

Fig. 6. Arduino UNO plus RedbearLab BLE shield (left), DFRobot Bluno Beetle BLE board (center), fully functional DIY SSSEP development board (right).

\section{Signal processing with OpenVibe}

The electrodes are placed on the scalp of the user according to the classical 10/20 international system. For instance, in our studies, electrodes were positioned on $\mathrm{C} 3$ and $\mathrm{C} 4$ location in order to detect brain signal responses to vibrations applied on right finger and left finger, respectively. Currently, the signal processing carried out using the OpenVibe software is dependent on the parameters manually supplied by the experimenter. For instance, as we can see on figure 7, a Butterworth Band Pass filter between a minimum and a maximum values can be easily implemented in OpenVibe. 


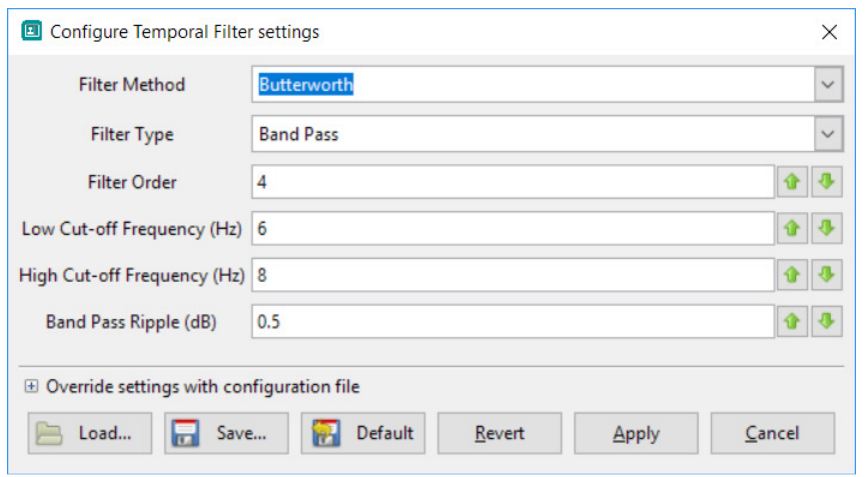

Fig. 7. Example of Butterworth Band Pass filter between 6 and $8 \mathrm{~Hz}$ usable in OpenVibe.

Being able to automatically filter the correct frequency to listen, according to the parameters indicated by the mobile application driven by the experimenter, should greatly accelerate the experiments and reduce the risk of error during BCI sessions.

\section{Conclusion and perspectives}

In this preliminary study, our goal was to propose a way to supervise SSSEP experiments with a mobile remote control application, in the context of a Brain-Computer Interaction, allowing to change dynamically the vibration that the user feels.

The technical part works correctly: the mobile application developed in our laboratory allows to quickly and easily choose a frequency (ex: $11 \mathrm{~Hz}$ ) and send this information, via Bluetooth, to an Arduino board (Bluno in our study), which drives a vibration motor, oscillating at the chosen frequency, under the finger or the wrist of the user. This allows a smooth manner to try various frequencies in a same session, without to stop, recalibrate and send again another chosen frequency during BCI experiments.

At the same time, this information (ex: $11 \mathrm{~Hz}$ ) is sent via UDP to the computer that manages the BCI scenario of the current experiment (OpenVibe in our study). We managed to recover this data in the OpenVibe scenario, thanks to a Python script, and it still remains to implement a dynamic filtering process of the signals, according to this parameter, by applying a bandpass filter (between 10 and $12 \mathrm{~Hz}$, in our example).

In the short term, we will try to improve the prototype in order to drive two vibration motors simultaneously. Thus, sending a simple command (ex: "LH:7RH:9") will allow to send at the same time two different orders, one for each vibrator motors ; example: Left Hand at $7 \mathrm{~Hz}$ and Right Hand at $9 \mathrm{~Hz}$. We are also planning to let the experimenter choose and generate some rest time between two vibrations, in order to find more easily the researched patterns in the cerebral waves. 
So this preliminary study for the use of physiological gating in a hybrid BCI shows that the technical part is correctly implemented. In the next step, we are planning to use more powerful vibrator, like C2-Tactors, for instance [36], for human experimentation and validation of the signal processing chain.

Obviously, this laboratory work is considered preparatory, and will not be deployed in a medical field where the electronic waves used to communicate between smartphones and PCs (Bluetooth in particular) could disrupt the proper functioning of the biological signal recorders.

However, we believe that the use of such supervisory tools for BCI should greatly facilitate the exploratory work of the experimenters, since seeking the right frequency to use for each patient requires a lot of time when done manually. The risk of error should also be reduced, ultimately, thanks to the semi-automatic generation of scripts used to detect the reflex responses produced by the human body for such somatosensory experiments.

\section{References}

1. Vidal, J.J.: Toward direct brain-computer communication. Annual review of Biophysics and Bioengineering 2:1, 157--180 (1973)

2. Leeb, R., Sagha, H., Chavarriaga, R., del R Millan, J.: Multimodal fusion of muscle and brain signals for a hybrid-BCI. In: Engineering in Medicine and Biology Society (EMBC), 2010 Annual International Conference of the IEEE, pp. $4343--4346$ (2010)

3. Luck, Steven J.:An Introduction to the Event-Related Potential Technique. The MIT Press (2005)

4. Colon E., Legraina,V., MourauxA.: Steady-state evoked potentials to study the processing of tactile and nociceptive somatosensory input in the human brain. Neurophysiologie Clinique/Clinical Neurophysiology (2012)

5. Luzheng B., Jinling L., Ke J., Ru L., Yili L.:A speed and direction-based cursor control system with P300 and SSVEP. Biomedical Signal Processing and Control 14, 26-133 (2014)

6. Trejo L.J. ${ }^{1}$, Rosipal R., Matthews B.:Brain-computer interfaces for 1-D and 2-D cursor control: designs using volitional control of the EEG spectrum or steady-state visual evoked potentials. IEEE Transactions on Neural Systems and Rehabilitation Engineering 14:2 (2006)

7. Faller J., Muller-Putz G., Schmalstieg D., Pfurtscheller G.: An application framework for controlling an avatar in a desktop-based virtual environment via a software SSVEP brain-computer interface. Presence: Teleoperators and Virtual Environments 19, 25--34 (2010)

8. Deng Z., Li X., Zheng K., Yao W.: A Humanoid Robot Control System with SSVEPbased Asynchronous Brain-Computer Interface. Robot 33:2, 129--135 (2011)

9. Prueckl R., Guger C.:A Brain-Computer Interface Based on Steady State Visual Evoked Potentials for Controlling a Robot. Cabestany J., Sandoval F., Prieto A., Corchado J.M. (eds) Bio-Inspired Systems: Computational and Ambient Intelligence. IWANN 2009. LNCS, vol. 5517. Springer, Heidelberg (2009)

10. Achic F., Montero J., Penaloza C., Cuellar F.:Hybrid BCI System to Operate an Electric Wheelchair and a Robotic Arm for Navigation and Manipulation Tasks. In: IEEE 
International Workshop on Advanced Robotics and its Social Impacts (ARSO) Shanghai (2016)

11. Vilic A., Kjaer T.W., Thomsen C.E., Puthusserypady S., Sorensen H.B.: DTU BCI speller: an SSVEP-based spelling system with dictionary support. In: 35th Annual International Conference of the IEEE EMBS, pp. 221--2215, Osaka (2013)

12. Combaz A., Chatelle C., Robben A., Vanhoof G., Goeleven A., Thijs V., Van Hulle M.M., Laureys S.: A Comparison of Two Spelling Brain-Computer Interfaces Based on Visual P3 and SSVEP in Locked-In Syndrome. Research Article (2013)

13. Punsawad Y., Wongsawat Y.: Multi-command SSAEP-based BCI system with training sessions for SSVEP during an eye fatigue state. IEEJ Transactions on Electrical and Electronic Engineering 12:S1, S72--S78 (2017)

14. Höhne J., Schreuder M., Blankertz B., Tangermann M.: A novel 9-class auditory ERP paradigm driving a predictive text entry system. Frontiers in Neuroscience 5:99 (2011)

15. Kim D.W., Hwang H.J., Lim J.H., Lee Y.H., Jung K.Y., Im C.H.: Classification of selective attention to auditory stimuli: Toward vision-free brain-computer interfacing. Journal of Neuroscience Methods 197:1, 180--185, (2011)

16. Guger C., Spataro R., Allison B.Z., Heilinger A., Ortner R., Cho W., La Bella V.: Complete Locked-in and Locked-in Patients: Command Following Assessment and Communication with Vibro-Tactile P300 and Motor Imagery Brain-Computer Interface Tools. Front Neurosci. 11: 251 (2017)

17. Kaufmann T., Herweg A., Kübler A.: Toward brain-computer interface based wheelchair control utilizing tactually-evoked event-related potentials. J. Neuroeng. Rehabil. $11: 7$ (2014)

18. Kim K.T., Suk H.I., Lee S.W.: Commanding a Brain-Controlled Wheelchair using Steady-State Somatosensory Evoked Potentials. IEEE Transactions on Neural Systems and Rehabilitation Engineering (2016)

19. Ahn S., Jun S.C.: Feasibility of hybrid BCI using ERD- and SSSEP- BCI. In: 12th International Conference on Control, Automation and Systems, pp. 2053-2056. Jeju Island (2012)

20. Treede R.D., Lorenz J., Baumgärtner U.: Clinical usefulness of laser-evoked potentials. Clinical Neurophysiology 33, 303-314 (2003)

21. Hsueh J.J., Jason Chen J.J., Shaw F.Z.: Distinct Somatic Discrimination Reflected by Laser-Evoked Potentials Using Scalp EEG Leads. J. Med. Biol. Eng. 36:4, 460--469 (2016)

22. Regan D.: Human Brain Electrophysiology: Evoked Potentials and Evoked Magentic Fields in Science and Medicine. Elsevier (1989)

23. Müller G.R., Neuper C., Pfurtscheller G.: Resonance-like frequencies of sensorimotor areas evoked by repetitive tactile stimulation. Biomed. Tech. 46, 186-190 (2001)

24. Müller-Putz G.R., Scherer R., Pfurtscheller G.: Steady-state somatosensory evoked potentials: Suitable brain signals for brain computer interfaces? IEEE Transactions on Neural Systems and Rehabilitation Engineering 14:1, 30--37 (2006)

25. Ahn S., Kim K., Jun S.C.: Steady-State Somatosensory Evoked Potential for BrainComputer Interface: Present and Future. Frontiers in Human Neuroscience 9:716 (2015)

26. Haegens S., Händel B.F., Jensen O.: Top-down controlled alpha band activity in somatosensory areas determines behavioral performance in a discrimination task. J. Neurosci. 31, 5197--5204. 
27. Breitwieser C., Kaiser V., Neuper C., Müller-Putz G.R.: Stability and distribution of steady-state somatosensory evoked potentials elicited by vibro- tactile stimulation. Med. Biol. Eng. Comput. 50, 347--357.

28. Yao L., Meng J., Zhang D., Sheng X., Zhu X.: Combining motor imagery with selective sensation toward a hybrid-modality BCI. IEEE Trans. Biomed. Eng. 61, 23042312 (2014)

29. Ahn S., Ahn M., Cho H., Jun S.C.: Achieving a hybrid brain- computer interface with tactile selective attention and motor imagery. J. Neural Eng. 11:6 (2014)

30. Chapman C.E.: Active versus passive touch: factors influencing the transmission of somatosensory signals to primary somatosensory cortex. Canadian Journal of Physiology and Pharmacology 72:5, 558--570 (1994)

31. Cheron G., Borenstein S.: Specific gating of the early somatosensory evoked potentials during active movement. Electroencephalography and Clinical Neurophysiology 67:6, 537--548 (1987)

32. Voisin, J., Rodrigues, E., Hétu, S., Jackson, P., Vargas, C., Malouin, F., Chapman, E., Mercier, C.: Modulation of the response to a somatosensory stimulation of the hand during the observation of manual actions. Experimental Brain Research 208, 11--19 (2010)

33. MIT App Inventor Extensions, BluetoothLE (edu.mit.appinventor.ble.aix), retrieved 14/02/2018, http://appinventor.mit.edu/extensions/

34. Andres Cotes, UDP Client Extension (co.com.dendritas.ClientUDP.aix), retrieved 14/02/2018, https://community.thunkable.com/t/udp-client-extension/5831

35. Texas Instruments, TI DRV2605 motor driver, retrieved 14/02/2018, http://www.ti.com/lit/ds/symlink/drv2605.pdf

36. C2 Tactors, provided by EAI (Engineering Acoustics, Inc.), www.eaiinfo.com, retrieved 14/02/2018,

http://bdml.stanford.edu/twiki/pub/Haptics/VibrationImplementation/C2_tactor.pdf 